\title{
Outcomes after pneumonectomy versus limited lung resection in adults with traumatic lung injury
}

\author{
Richelle L. Homo ${ }^{1}$ D $\cdot$ Areg Grigorian ${ }^{1} \cdot$ Michael Lekawa ${ }^{1} \cdot$ Matthew Dolich ${ }^{1} \cdot$ Catherine M. Kuza $^{2}$. \\ Andrew R. Doben ${ }^{3} \cdot$ Ronald Gross ${ }^{3} \cdot$ Jeffry Nahmias ${ }^{1}$
}

Received: 22 May 2019 / Accepted: 14 February 2020 / Published online: 21 February 2020

(C) Italian Society of Surgery (SIC) 2020

\begin{abstract}
Pneumonectomy after traumatic lung injury (TLI) is associated with shock, increased pulmonary vascular resistance, and eventual right ventricular failure. Historically, trauma pneumonectomy (TP) mortality rates ranged between 53 and $100 \%$. It is unclear if contemporary mortality rates have improved. Therefore, we evaluated outcomes associated with TP and limited lung resections (LLR) (i.e., lobectomy and segmentectomy) and aimed to identify predictors of mortality, hypothesizing that TP is associated with greater mortality versus LLR. We queried the Trauma Quality Improvement Program (2010-2016) and performed a multivariable logistic regression to determine the independent predictors of mortality in TLI patients undergoing TP versus LLR. TLI occurred in 287,276 patients. Of these, 889 required lung resection with $758(85.3 \%)$ undergoing LLR and 131 (14.7\%) undergoing TP. Patients undergoing TP had a higher median injury severity score (26.0 vs. 24.5, $p=0.03)$ but no difference in initial median systolic blood pressure (109 vs. $107 \mathrm{mmHg}, p=0.92)$ compared to LLR. Mortality was significantly higher for TP compared to LLR $(64.9 \%$ vs $27.2 \%, p<0.001)$. The strongest independent predictor for mortality was undergoing TP versus LLR (OR 4.89, CI 3.18-7.54, $p<0.001$ ). TP continues to be associated with a higher mortality compared to LLR. Furthermore, TP is independently associated with a fivefold increased risk of mortality compared to LLR. Future investigations should focus on identifying parameters or treatment modalities that improve survivability after TP. We recommend that surgeons reserve TP as a last-resort management given the continued high morbidity and mortality associated with this procedure.
\end{abstract}

Keywords Traumatic lung injury $\cdot$ Trauma pneumonectomy $\cdot$ Limited lung resections

\section{Introduction}

Thoracic trauma accounts for $20-60 \%$ of all trauma injuries and is a major contributor to trauma-related mortality [1-4]. The true incidence of traumatic lung injury (TLI) is unknown, as many patients with thoracic trauma die prior to

Richelle L. Homo

richellelh@gmail.com

1 Division of Trauma, Burns and Surgical Critical Care, Department of Surgery, University of California, Irvine Medical Center, 333 The City Blvd West, Suite 1600, Orange, CA 92868-3298, USA

2 Department of Anesthesiology, University of Southern California, Los Angeles, CA, USA

3 Department of Surgery, Baystate Medical Center Affiliate of Tufts University School of Medicine, Springfield, MA, USA arrival at the hospital [5, 6]. Among those presenting with thoracic trauma, pulmonary contusion is found in $30-75 \%$, and pneumothorax and/or hemothorax in $20 \%$ of patients $[2,7]$. Typically, most patients presenting with chest trauma require less invasive interventions, such as chest tube placement; however, in rare and severe cases, surgical resection may be necessary. TLI requiring surgical lung resection and the extent of lung resected are associated with increased morbidity and morality [8]. Overall, less than $0.1 \%$ of patients with chest trauma require any type of lung resection; however, patients who do undergo lung resection have significantly higher mortality rates [9-11].

Mortality rates for lung resection vary based on the extent of surgical resection. Rates are 10-22\% for a wedge resection, $27-70 \%$ for a lobectomy, and $53-100 \%$ for a trauma pneumonectomy (TP) $[9,12,13]$. Although less extensive lung resection techniques, such as wedge resections, may result in lower mortality rates, they may not always be 
feasible, especially when patients present with more severe lung injuries that are not amenable to this technique. More devastating injuries such as major hilar or bronchial injuries warrant more extensive lung resections, which are also associated with longer operative times and increased blood loss $[9,12,14]$. Previous studies comparing trauma patient outcomes associated with varying degrees of lung resection demonstrated that more extensive lung resections were associated with increased complications, and that the extent of lung resection is an independent predictor of mortality, even after adjusting for injury severity $[9,14]$.

The purpose of this study was to evaluate if in-hospital mortality in patients with TLI undergoing TP and limited lung resections (LLR) (i.e., lobectomy and segmentectomy) have improved compared to historical reports by analyzing data collected from the Trauma Quality Improvement Program (TQIP) database. We hypothesized that TLI patients undergoing TP had higher mortality rates compared to those undergoing LLR. Additionally, we performed a contemporary analysis of TLI to determine if mortality rates after TP have improved over time and identified independent risk factors associated with mortality after TLI.

\section{Methods}

This retrospective cohort database study was approved by the Institutional Review Board. The manuscript adheres to the relevant guidelines outlined in the Strengthening the Reporting of Observational Studies in Epidemiology (STROBE) statement [15].

\section{Study population}

The TQIP of the American College of Surgeons is a quality improvement vehicle where Level I and II trauma centers can electively contribute data and in turn, receive an annual confidential report benchmarking outcomes compared to other trauma centers [16]. We queried the TQIP database between 2010 and 2016. The study included all trauma patients $\geq 18$ years old presenting with lung injuries (i.e., laceration, contusion, pneumothorax, hemothorax, hemopneumothorax, etc.) defined by the International Classification of Diseases version 9 (ICD-9) diagnosis codes: 860-860.5 and 861.2-861.32 who underwent surgical management, including pneumonectomy, lobectomy, or segmentectomy that were identified using the ICD-9 procedure codes: TP $(32.5,32.59)$, lobectomy $(32.41,32.49)$, and segmentectomy $(32.3,32.39)$. There was no exclusion criteria. We also identified patients who underwent damage control surgery (DCS) with the ICD-9 procedure code for re-incision of recent thoracotomy site (34.03) to determine if there was a difference in DCS rates between TP and LLR, and if DCS affected survival in TP patients.

Patient demographic information was collected in addition to injury-related data, median systolic blood pressure (SBP), Glasgow Coma Scale (GCS) score on arrival, concomitant traumatic injuries, and pre-hospital comorbidities. Pre-hospital comorbidities included congestive heart failure (CHF), smoking, diabetes, hypertension, and chronic obstructive pulmonary disease (COPD). The injury profile included the injury severity score (ISS), mechanism of injury, and comorbid injuries to the spine, brain, pelvis, heart, stomach, small and large bowel, pancreas, liver, spleen, and kidney.

\section{Outcomes}

The primary outcome was in-hospital mortality. Secondary outcomes included: total hospital length of stay (LOS), intensive care unit (ICU) LOS, ventilator days, and complications such as acute respiratory distress syndrome (ARDS), cardiac arrest, and pneumonia.

\section{Statistical analysis}

We compared the outcomes between patients undergoing TP versus LLR. Additionally, we examined the trends over time of trauma patients undergoing TP and LLR between 2010 and 2016. A power analysis was performed and sample size determination was made to guarantee an adequate significance of the results obtained from this study. Sample size estimates were for Type I error of 5\% $(\alpha=0.05)$ and power $(1-\beta)$ of $90 \%$. The effect size was determined by estimating mortality as 55\% in the TP group and $40 \%$ for the LLR group $[9,12,13]$. It was determined that a sample size of at least 462 patients split between the two groups would be needed to generate sufficient statistical power to detect a difference. Descriptive statistics were performed for all variables. A Mann-Whitney $U$ test was used to compare continuous variables and chi-square was used to compare categorical variables. Categorical data were reported as percentages, and continuous data were reported as medians with interquartile range or means with standard deviation.

We performed an analysis to identify predictors of morality in TLI patients. The magnitude of the association between predictor variables and mortality was measured using a univariable logistic regression model. These variables were chosen based on a review of the literature, and included age, surgery type (TP vs. LLR), cardiac injury, traumatic brain injury (TBI), ISS, and pulmonary complications (i.e., pneumonia, ARDS) [8,9]. Covariates were then controlled for using a hierarchical multivariable logistic regression model. This was reported with an odds ratio (OR) and $95 \%$ confidence intervals (CI). All $p$ values were 
two sided, with a statistical significance level of $<0.05$. All statistical analyses were performed with IBM SPSS Statistics for Windows, Version 24. (Armonk, NY: IBM Corp).

\section{Results}

\section{TLI patient demographics and characteristics}

Of the 287,276 adult patients presenting with TLI, 889 $(0.31 \%)$ underwent a lung resection, with $758(85.3 \%)$ undergoing LLR and 131 (14.7\%) undergoing a TP. The TP and LLR groups had a similar median age (28 years old, $p=0.99)$ and both groups were predominately males (TP: 84.7\%; LLR: $87.5 \%, p=0.39)$. There were no differences in prehospital comorbidities, except for smoking (6.9\% vs $16.6 \%, p<0.01)$ and COPD (0.8\% vs $5.4 \%, p=0.02)$ which were lower in the TP group (Table 1). Patients who underwent a TP had a higher median ISS (26.0 vs. $24.5, p=0.03$ ), lower median GCS on admission (4.5 vs $13.0, \mathrm{p}<0.001)$, but no difference in initial median SBP (109 vs. $107 \mathrm{mmHg}$, $p=0.92$ ). The most common mechanism of injury in both groups was gunshot wound (TP: $48.1 \%$; LLR: $46.2 \%$, $p=0.69)$.

\section{Primary and secondary outcomes}

The overall mortality of those requiring lung resection was $32.7 \%$. Mortality was significantly greater in those undergoing TP compared to LLR (64.9\% vs $27.2 \%, p<0.001)$. Patients who underwent TP had shorter median hospital LOS ( 2.5 vs. 13 days, $p<0.001)$ and median ventilator days compared to those who underwent LLR ( 2 vs. $4, p=0.03$ ) (Table 2). The median ICU LOS was similar between TP and LLR groups ( 4 vs. 7 days, $p=0.05$ ). There were significantly more patients who had cardiac arrest (TP: $31.3 \%$; LLR: $12.7 \%, p<0.001)$ in the TP compared with the LLR group. There was no difference in the number of patients who developed ARDS (TP: 7.6\%; LLR 9.4\%, $p=0.52$ ) between the two groups. Fewer patients in the TP group developed pneumonia compared with the LLR group (TP: 8.4\%; LLR: $17.0 \%, p=0.01)$. There was no difference in the number of patients who underwent DCS between both groups (TP: 7.6\%; LLR: $7.9 \%, p=0.91$ ).

\section{Trends of lung surgeries in TLI patients performed over time}

The overall rate of lung resections did not change from $0.28 \%$ in 2010 to $0.31 \%$ in 2016 ( $p=0.56$ ) (Fig. 1). The rate of TP among those who required lung resection remained statistically similar from $22.0 \%$ in 2010 to $12.2 \%$ in 2016 $(p=0.16)$ (Fig. 2).
Table 1 Demographics of trauma patients presenting with lung injury and undergoing lung resection between 2010 and 2016

\begin{tabular}{|c|c|c|c|}
\hline Characteristic & $\begin{array}{l}\text { LLR } \\
(n=758)\end{array}$ & $\begin{array}{l}\text { Pneumonectomies } \\
(n=131)\end{array}$ & $p$ value \\
\hline Age, year, median (IQR) & $28(20)$ & $28(17)$ & 0.99 \\
\hline Male, $n(\%)$ & $663(87.5 \%)$ & $111(84.7 \%)$ & 0.39 \\
\hline ISS, median (IQR) & $24.5(18)$ & $26.0(22)$ & 0.03 \\
\hline $\begin{array}{l}\text { SBP, mmHg, median } \\
\text { (IQR) }\end{array}$ & $107.0(49)$ & $109.0(53)$ & 0.92 \\
\hline GCS, median (IQR) & $13.0(12)$ & $4.5(11)$ & $<0.001$ \\
\hline \multicolumn{4}{|l|}{ Mechanism, $n(\%)$} \\
\hline Stab wound & $80(10.6 \%)$ & $7(5.3 \%)$ & 0.06 \\
\hline Gunshot wound & $350(46.2 \%)$ & $63(48.1 \%)$ & 0.69 \\
\hline $\begin{array}{l}\text { Motor vehicle } \\
\text { accident }\end{array}$ & $107(14.1 \%)$ & $17(13.0 \%)$ & 0.73 \\
\hline Pedestrian struck & $17(2.2 \%)$ & $3(2.3 \%)$ & 0.97 \\
\hline Bicycle accident & $7(0.9 \%)$ & $1(0.8 \%)$ & 0.86 \\
\hline Fall & $35(4.6 \%)$ & $8(6.1 \%)$ & 0.46 \\
\hline Suicide & $84(11.1 \%)$ & $19(14.5 \%)$ & 0.26 \\
\hline \multicolumn{4}{|l|}{ Comorbidities, $n(\%)$} \\
\hline $\begin{array}{l}\text { Congestive heart } \\
\text { failure }\end{array}$ & $1(0.1 \%)$ & $1(0.8 \%)$ & 0.16 \\
\hline Smoker & $126(16.6 \%)$ & $9(6.9 \%)$ & 0.004 \\
\hline Diabetes mellitus & $18(2.4 \%)$ & $1(0.8 \%)$ & 0.24 \\
\hline Hypertension & $55(7.3 \%)$ & $5(3.8 \%)$ & 0.15 \\
\hline COPD & $41(5.4 \%)$ & $1(0.8 \%)$ & 0.02 \\
\hline \multicolumn{4}{|l|}{ Injuries, $n(\%)$} \\
\hline $\begin{array}{l}\text { Traumatic brain } \\
\text { injury }\end{array}$ & $114(15.0 \%)$ & $29(22.1 \%)$ & 0.04 \\
\hline Spine & $217(28.6 \%)$ & $28(21.4 \%)$ & 0.09 \\
\hline Pelvis & $77(10.2 \%)$ & $10(7.6 \%)$ & 0.37 \\
\hline Upper extremity & $182(24.0 \%)$ & $37(28.2 \%)$ & 0.30 \\
\hline Lower extremity & $69(9.1 \%)$ & $15(11.5 \%)$ & 0.40 \\
\hline Cardiac & $71(9.4 \%)$ & $19(14.5 \%)$ & 0.07 \\
\hline Stomach & $37(4.9 \%)$ & $4(3.1 \%)$ & 0.36 \\
\hline Small intestine & $33(4.4 \%)$ & $4(3.1 \%)$ & 0.49 \\
\hline Colorectal & $43(5.7 \%)$ & $6(4.6 \%)$ & 0.61 \\
\hline Pancreas & $11(1.5 \%)$ & $0(0.0 \%)$ & 0.17 \\
\hline Liver & $161(21.2 \%)$ & $26(19.8 \%)$ & 0.72 \\
\hline Spleen & $96(12.7 \%)$ & $19(14.5 \%)$ & 0.56 \\
\hline Kidney & $62(8.2 \%)$ & $6(4.6 \%)$ & 0.15 \\
\hline
\end{tabular}

Significant $p$-values are in bold

$L L R$ limited lung resection (lobectomy/segmentectomy), $I Q R$ interquartile range, ISS injury severity score, $S B P$ systolic blood pressure, $G C S$ Glasgow Coma Scale, COPD chronic obstructive lung disease

\section{Mortality risk factors in TLI patients}

In a multivariable logistic regression model, the strongest independent risk factor for mortality was undergoing TP versus LLR (OR 4.89, CI 3.18-7.54, $p<0.001$ ) followed by ISS $\geq 25$ (OR 3.09, CI 2.23-4.29, $p<0.001$ ) (Table 3). Other 
Table 2 Clinical outcomes of trauma patients presenting with lung injury and undergoing lung resection between 2010 and 2016

\begin{tabular}{lclc}
\hline Outcome & $\begin{array}{l}\text { LLR } \\
(n=758)\end{array}$ & $\begin{array}{l}\text { Pneumonectomy } \\
(n=131)\end{array}$ & $p$ value \\
\hline LOS, days, median (IQR) & $13(16)$ & $2.5(14)$ & $<\mathbf{0 . 0 0 1}$ \\
ICU, days, median (IQR) & $7(14)$ & $4(18)$ & 0.05 \\
Ventilation, days, median & $4(11)$ & $2(8)$ & $\mathbf{0 . 0 3}$ \\
(IQR) & & & \\
Damage control surgery & $60(7.9 \%)$ & $10(7.6 \%)$ & 0.91 \\
Complications & & & \\
ARDS & $71(9.4 \%)$ & $10(7.6 \%)$ & 0.52 \\
Cardiac arrest & $96(12.7 \%)$ & $41(31.3 \%)$ & $<\mathbf{0 . 0 0 1}$ \\
Pneumonia & $129(17.0 \%)$ & $11(8.4 \%)$ & $\mathbf{0 . 0 1}$ \\
Mortality, $n(\%)$ & $206(27.2 \%)$ & $85(64.9 \%)$ & $<\mathbf{0 . 0 0 1}$ \\
\hline
\end{tabular}

Significant $p$-values are in bold

LLR limited lung resection (lobectomy/segmentectomy), LOS length of stay, IQR interquartile range, ICU intensive care unit, $A R D S$ acute respiratory distress syndrome

independent risk factors for mortality include: age $\geq 65$ years (OR 2.97, CI 1.44-6.10, $p<0.01$ ), cardiac injury (OR 2.26, CI 1.38-3.70, $p<0.01$ ), TBI (OR 1.75, CI 1.15-2.67, $p<0.01$ ), and ARDS (OR 2.59, CI 1.45-4.62, $p=0.001$ ).

\section{Discussion}

To our knowledge, this is the largest database study in the United States that examined TLI in adult patients, compared mortality rates between different surgical interventions, and reported trends in surgical procedures over time. Importantly, this retrospective analysis using seven years of data from TQIP demonstrated that TP continues to have a significantly higher mortality than that of LLR among patients with TLI requiring surgical intervention. Furthermore, TP was identified as the strongest risk factor for mortality in TLI patients. Although we reported that patients who underwent TP had shorter LOS and ventilator days compared to LLR, these patients had higher in-hospital mortality rates and most likely died early in their hospital course, thereby skewing these data. In addition, the current mortality rate of TP appears relatively unchanged compared to previous reports, thereby reinforcing the need for future research on this highly lethal operation.

The prevalence of lung resections in thoracic trauma was previously reported to be $0.08 \%$ with higher rates after penetrating (1.3\%) compared with blunt injury $(0.03 \%)$ [9]. Huh et al. performed a single-center retrospective review between 1984 and 1999 and reported mortality rates of $69.7 \%$ for TP and $35.0 \%$ for lobectomy [11]. Similarly, a review of 669 TLI patients undergoing lung resection identified in the National Trauma Database by Martin et al. reported mortality rates of $62 \%$ for TP and 38\% for lobectomy. Even in isolated TLI, the mortality rate for TP was $53 \%$ and $27 \%$ for lobectomy [9]. More than a decade after these reports, we demonstrated that patients undergoing TP had a mortality rate of $65 \%$ and $27 \%$ for LLR.

Although the mortality rate for TP remains high and similar to historical reports, our analysis revealed that trends for TP and overall lung resection in TLI patients remained stable between 2010 and 2016. The extent of lung resection is associated with longer operative times, increased blood loss, and higher perioperative morbidity and mortality $[9,12,14]$. For example, pulmonary tractotomy preserves lung tissue in patients requiring emergent thoracotomy and presenting with penetrating or blunt through-and-through pulmonary parenchyma injury not involving hilar structures [11, 17, 18]. For patients with hilar or irreparable main bronchus injuries where TP may not be avoided, rapid stapled pneumonectomy may improve outcomes, as the survival of the patient may be related to the rapidity by which the hilum is
Fig. 1 Overall trend of lung resection in trauma patients with lung injury

\section{Overall Rate of Lung Resections}

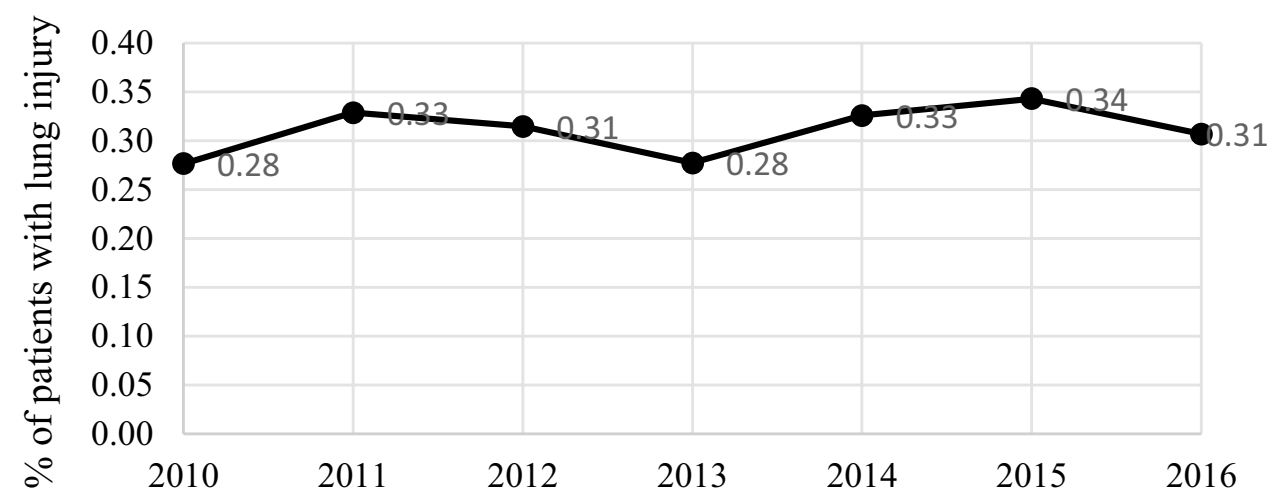

- Total Lung Resections 
Fig. 2 Lung resection trends in trauma patients with lung injury who required lung resection between 2010 and 2016

\section{Lung Resection Trends}

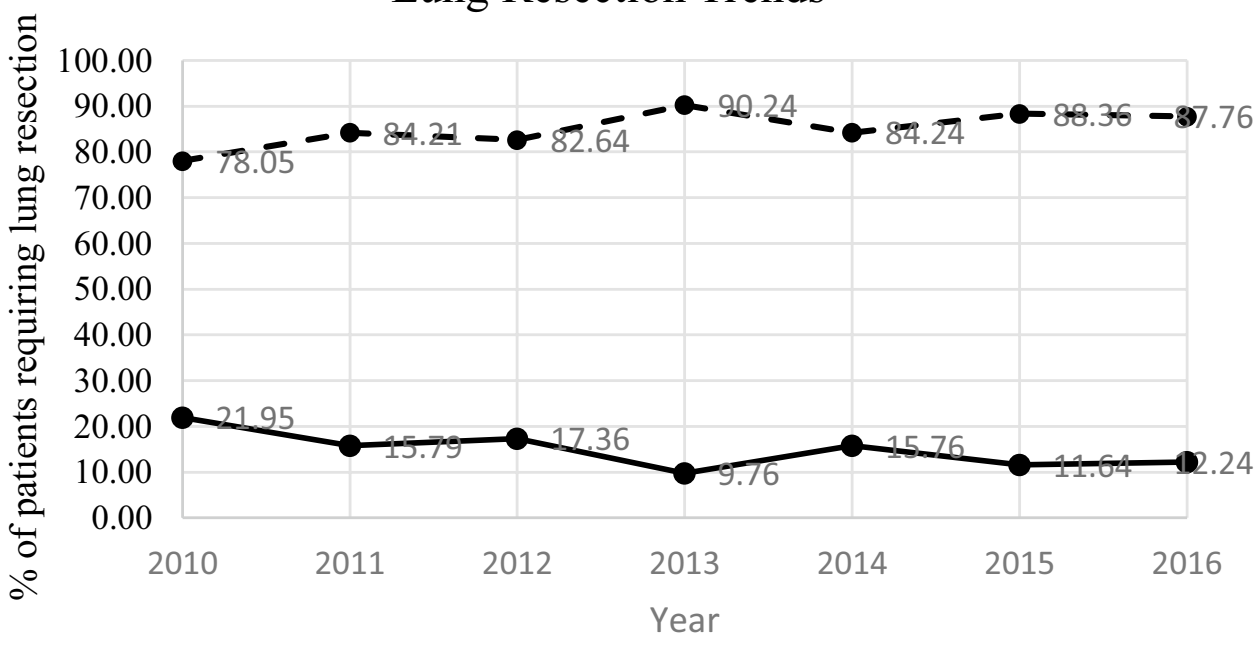

$\longrightarrow$ Trauma Pneumonectomy - - Limited Lung Resection
Table 3 Multivariable logistic regression analysis for risk of mortality in trauma patients presenting with lung injury

\begin{tabular}{lllr}
\hline Risk factor & OR & CI & $p$ value \\
\hline Pneumonectomy vs. LLR & 4.89 & $3.18-7.54$ & $<\mathbf{0 . 0 0 1}$ \\
Age $\geq$ 65 years & 2.97 & $1.44-6.10$ & $\mathbf{0 . 0 0 3}$ \\
ISS $\geq 25$ & 3.09 & $2.23-4.29$ & $<\mathbf{0 . 0 0 1}$ \\
Cardiac injury & 2.26 & $1.38-3.70$ & $\mathbf{0 . 0 0 1}$ \\
Traumatic brain injury & 1.75 & $1.15-2.67$ & $\mathbf{0 . 0 0 9}$ \\
Complication & & & \\
$\quad$ ARDS & 2.59 & $1.45-4.62$ & $\mathbf{0 . 0 0 1}$ \\
\multicolumn{1}{l}{ Pneumonia } & 0.20 & $0.12-0.36$ & $<\mathbf{0 . 0 0 1}$ \\
\hline
\end{tabular}

Significant $p$-values are in bold

$L L R$ limited lung resection (lobectomy/segmentectomy), ISS injury severity score, $A R D S$ acute respiratory distress syndrome

compressed and the lung is resected [18]. Another technique reported in other studies is twisting the pulmonary hilum in central lung injuries to gain rapid control of hemorrhage and postpone pneumonectomy $[11,19]$. Nonetheless, this study emphasizes the clinical implication that lung-sparing techniques should be performed whenever possible.

The use of DCS techniques have successfully improved survival rates in severe abdominal, vascular, and orthopedic trauma, and have recently been applied to those with thoracic injuries [17, 20, 21]. However, there are few singlecenter studies with small patient sample sizes that describe the impact of DCS on outcomes in patients with TLI [19, 21, 22]. Studies that report lower overall mortality rates $(\sim 24 \%)$ associated with DCS still find the highest mortality rates in patients who undergo TP (up to 67\%), compared to less invasive surgeries such as lobectomy or wedge resection [19,
21]. This highlights that the cause of death after TP is different than the cause of death after DCS for abdominal hemorrhage and it is important for the clinician to understand that the mortality associated with TP may not necessarily be related to the procedure alone.

Previous studies that explored surgical outcomes after TP have attributed the cause of mortality to concomitant intraabdominal or severe head injuries, greater overall ISS on presentation, and uncontrolled hemorrhage leading to refractory shock $[8,9,12]$. Martin et al. found that the lowest survival rates in TP patients were seen in those who presented with hypotension, ISS $\geq 15$, and low GCS [9]. Our study supports these findings, demonstrating a threefold increase in mortality in patients with ISS $\geq 25$ and a nearly twofold increase in those with TBI. Furthermore, earlier studies have found a stepwise increase in mortality with extent of lung resection [8, 9, 19, 23]. Our study corroborates these findings and further demonstrates a fivefold increased risk of mortality with TP compared to LLR after controlling for significant risk factors for mortality in trauma patients such as ISS, cardiac injury, and complications (i.e., ARDS).

Poor outcomes associated with TP may also be in part due to cardiopulmonary stress. Previous animal studies have demonstrated that pneumonectomy results in an acute increase in pulmonary vascular resistance (PVR) due to an increase in pulmonary artery pressure without significant change in cardiac index [24]. This is thought to be mediated by cytokine release associated with hemorrhagic shock and blood product transfusion after lung resection [25-27]. This causes decreased compliance and increased afterload in the right ventricle $(\mathrm{RV})$, which leads to a rapid onset of $\mathrm{RV}$ failure, decreased cardiac output, tissue hypoxia, refractory 
shock, and death. Indeed, there were significantly more patients who developed cardiac arrest in the TP group than the LLR group. These physiological changes may be exaggerated in trauma patients, who are already suffering from other extreme physiologic derangements.

Therefore, interventions that minimize postoperative increases in PVR may improve survivability in TP patients. Inhaled nitric oxide (iNO) has been demonstrated to selectively dilate the pulmonary vasculature, decrease PVR, and improve oxygenation and ventilation perfusion with minimal effects on systemic hemodynamics [24, 26, 28, 29]. A blinded randomized controlled trial on sheep with hemorrhagic shock demonstrated that iNO could decrease right ventricle afterload, while preserving biventricular function and, therefore, may be a useful adjunct for patients undergoing TP [28]. Future large prospective trials using iNO or other novel treatments for humans undergoing TP are needed to try and mitigate the persistently high mortality associated with this surgery.

There are several limitations to this study including those inherent to a retrospective database study such as entry errors and selection and reporting bias. Our data were restricted to fields available in the TQIP database. For example, lobectomy and segmentectomy were grouped together as LLR, while they have been analyzed separately in previous studies. Missing pertinent data fields include the indication for operation, extent of injury (i.e., American Association for the Surgery of Trauma (AAST) lung injury grade), surgical technique (i.e., hilar twist, clamp resection, staple resection), intraoperative physiology, intraoperative surgical findings (i.e., cardiac injury or great vessel injury), surgical duration, intraoperative blood loss, percentage of lung resected, and whether ischemic pre-conditioning was utilized. In addition, we were unable to include perioperative data such as fluid resuscitation, ventilatory management, and hemodynamic support with vasopressors. The timing of death and cause of mortality were also not available. Furthermore, we were unable to determine if patients who went for re-incision of recent thoracotomy site were planned or unplanned cases returning to the operating room. Lastly, we were unable to evaluate the laterality in this patient population, which may change outcomes as a right pneumonectomy has been associated with a fivefold increase in mortality compared to a left pneumonectomy in elective thoracic lung resections [23, 30, 31]. These limitations could ideally be addressed in a prospective study or with data containing the aforementioned missing information in future versions of TQIP.

In conclusion, TP continues to be associated with a higher mortality compared to LLR. Furthermore, the mortality rate appears unchanged compared to previous historical reports. Future investigations should focus on identifying alternative interventions to manage TLI to avoid performing TP and/or pharmacologic interventions that may mitigate the deleterious cardiopulmonary effects of TP. Until then, we recommend that surgeons reserve TP as a last-resort management given the continued high morbidity and mortality associated with this procedure.

Funding This research did not receive any specific grant from funding agencies in the public, commercial, or not-for-profit sectors.

\section{Compliance with ethical standards}

Conflict of interest The authors report no conflicts of interest, financial or otherwise.

Research involving Human Participants and/or Animals This article does not contain any studies with human participants or animals performed by any of the authors.

Informed consent Informed consent is not required for this type of study.

\section{References}

1. Probst C, Pape HC, Hildebrand F et al (2009) 30 years of polytrauma care: an analysis of the change in strategies and results of 4849 cases treated at a single institution. Injury 40:77-83. https ://doi.org/10.1016/j.injury.2008.10.004

2. Kulshrestha P, Munshi I, Wait R (2004) Profile of chest trauma in a level I trauma center. J Trauma Inj Infect Crit Care 57:576-581. https://doi.org/10.1097/01.TA.0000091107.00699.C7

3. Hunt PA, Greaves I, Owens WA (2006) Emergency thoracotomy in thoracic trauma-a review. Injury 37:1-19. https://doi. org/10.1016/j.injury.2005.02.014

4. Platz JJ, Fabricant L, Norotsky M (2017) Thoracic trauma: injuries, evaluation, and treatment. Surg Clin North Am 97:783-799. https://doi.org/10.1016/j.suc.2017.03.004

5. Clarke DL, Quazi MA, Reddy K, Thomson SR (2011) Emergency operation for penetrating thoracic trauma in a metropolitan surgical service in South Africa. J Thorac Cardiovasc Surg 142:563568. https://doi.org/10.1016/j.jtcvs.2011.03.034

6. Petrone P, Asensio JA (2009) Surgical management of penetrating pulmonary injuries. Scand J Trauma Resusc Emerg Med 17:8. https://doi.org/10.1186/1757-7241-17-8

7. Daurat A, Millet I, Roustan JP et al (2016) Thoracic trauma severity score on admission allows to determine the risk of delayed ARDS in trauma patients with pulmonary contusion. Injury 47:147-153. https://doi.org/10.1016/j.injury.2015.08.031

8. Matsushima K, Aiolfi A, Park C et al (2017) Surgical outcomes after trauma pneumonectomy: revisited. J Trauma Acute Care Surg 82:927-932. https://doi.org/10.1097/TA.0000000000001416

9. Martin MJ, McDonald JM, Mullenix PS et al (2006) Operative management and outcomes of traumatic lung resection. J Am Coll Surg 203:336-344. https://doi.org/10.1016/j.jamcollsur g.2006.05.009

10. Stewart KC, Urschel JD, Nakai SS et al (1997) Pulmonary resection for lung trauma. Ann Thorac Surg 63:1587-1588. https://doi. org/10.1016/S0003-4975(97)00442-6

11. Huh J, Wall MJ, Estrera AL et al (2003) Surgical management of traumatic pulmonary injury. Am J Surg 186:620-624. https://doi. org/10.1016/j.amjsurg.2003.08.013 
12. Cothren C, Moore EE, Biffl WL et al (2002) Lung-sparing techniques are associated with improved outcome compared with anatomic resection for severe lung injuries. J Trauma Inj Infect Crit Care 53:483-487. https://doi.org/10.1097/00005373-20020 9000-00015

13. Velmahos GC, Gervasini A, Petrovick L et al (2006) Routine Repeat Head CT for Minimal Head Injury is Unnecessary. J Trauma. https://doi.org/10.1097/01.ta.0000203546.14824.0d

14. Karmy-Jones R, Jurkovich GJ, Shatz DV et al (2001) Management of traumatic lung injury: a Western Trauma Association Multicenter review. J Trauma 51:1049-1053. https://doi. org/10.1097/00005373-200112000-00004

15. von Elm E, Altman DG, Egger M et al (2014) The strengthening the reporting of observational studies in epidemiology (STROBE) statement: guidelines for reporting observational studies. Int J Surg 12:1495-1499. https://doi.org/10.1016/j.ijsu.2014.07.013

16. Shafi S, Nathens AB, Cryer HG et al (2009) The trauma quality improvement program of the American College of Surgeons Committee on Trauma. ACS 209:521-530.e1. https://doi. org/10.1016/j.jamcollsurg.2009.07.001

17. Rotondo MF, Bard MR (2004) Damage control surgery for thoracic injuries. Injury 35:649-654. https://doi.org/10.1016/j.injur y.2004.03.002

18. Roberts DJ, Bobrovitz N, Zygun DA et al (2016) Indications for use of damage control surgery in civilian trauma patients. A content analysis and expert appropriateness rating study. Ann Surg 263:1018-1027. https://doi.org/10.1097/SLA.000000000000134 7

19. Garcia A, Martinez J, Rodriguez J et al (2015) Damage-control techniques in the management of severe lung trauma. J Trauma Acute Care Surg 78:45-50. https://doi.org/10.1097/TA.00000 00000000482

20. Rotondo MF, Zonies DH (1997) The damage control sequence and underlying logic. Surg Clin North Am 77:761-777. https:// doi.org/10.1016/S0039-6109(05)70582-X

21. O'Connor JV, DuBose JJ, Scalea TM (2014) Damage-control thoracic surgery: Management and outcomes. J Trauma Acute Care Surg 77:660-665. https://doi.org/10.1097/TA.000000000000045 1

22. Mackowski M, Barnett R, Harbrecht B et al (2013) Damage control in thoracic trauma. J Orthop Traumatol Rehabil 6:13. https:// doi.org/10.4103/0975-7341.118733
23. Wahi R, McMurtrey MJ, DeCaro LF et al (1989) Determinants of perioperative morbidity and mortality after pneumonectomy. Ann Thorac Surg 48:33-37. https://doi.org/10.1016/00034975(89)90172-0

24. Cryer HG, Mavroudis C, Yu J et al (1990) Shock, transfusion, and pneumonectomy: death is due to right heart failure and increased pulmonary vascular resistance. Ann Surg 212:197-201. https:// doi.org/10.1097/00000658-199008000-00014

25. Nurozler F, Argenziano M, Ginsburg ME (2001) Nitric oxide usage after posttraumatic pneumonectomy. Ann Thorac Surg 71:364-366. https://doi.org/10.1016/S0003-4975(00)02225-6

26. Maxey TS, Fernandez LG, Reece TB et al (2006) Endothelial nitric oxide synthase is essential for postpneumonectomy compensatory vasodilation. Ann Thorac Surg 81:1234-1238. https:// doi.org/10.1016/j.athoracsur.2005.11.049

27. Della Rocca G, Coccia C (2005) Nitric oxide in thoracic surgery. Minerva Anestesiol 71:313-318

28. Lubitz A, Sjoholm L, Goldber A et al (2017) Acute right heart failure after hemorrhagic shock and trauma pneumonectomy-a management approach: a blinded randomized controlled animal trial using inhaled nitric oxide Andrea. J Trauma Acute Care Surg 82:243-251. https://doi.org/10.1080/10937404.2015.10516 11.INHALATION

29. Halonen-Watras J, O'Connor J, Scalea TM (2011) Traumatic pneumonectomy: a viable option for patients in extremis. Am Surg 77:493-497. https://doi.org/10.1518/001872008X288600

30. Darling GE, Abdurahman A, Yi QL et al (2005) Risk of a right pneumonectomy: role of bronchopleural fistula. Ann Thorac Surg 79:433-437. https://doi.org/10.1016/j.athoracsur.2004.07.009

31. Bernard A, Deschamps C, Allen MS et al (2001) Pneumonectomy for malignant disease: factors affecting early morbidity and mortality. J Thorac Cardiovasc Surg 121:1076-1082. https://doi. org/10.1067/mtc.2001.114350

Publisher's Note Springer Nature remains neutral with regard to jurisdictional claims in published maps and institutional affiliations. 